\title{
OBSERVATIONS OF PLANETARY-NEBULA SPECTRA \\ IN THE NEAR ULTRAVIOLET
}

\author{
E. B. Kostuakova \\ (Sternberg State Astronomical Institute, Moscow, U.S.S.R.)
}

\begin{abstract}
A BST RACT
Several dozen spectrograms of about 20 nebulae were obtained with the quartz spectrograph, placed at the Cassegrain focus of the $125-\mathrm{cm}$ parabolic reflector of the Sternberg State Astronomical Institute Crimean Station. Nebulae with intense Balmer continuum were selected. Nearly half of them had not been studied before. Reductions were made for the nuclei spectra, for the differential atmospheric extinction and for the interstellar absorption. The A0 V stars were taken as absolute intensity standards. The work is not yet finished, but the results will be received in a short time.
\end{abstract}

Osterbrock and O'Dell (eds.), Planetary Nebulae, 62. (C) I.A.U. 